\title{
BMJ Open Methods for the health technology assessment of complex interventions: a protocol for a scoping review
}

\author{
Abdolvahab Baghbanian (D) , Tracy Merlin, Drew Carter, Shuhong Wang
}

To cite: Baghbanian A, Merlin T, Carter D, et al. Methods for the health technology assessment of complex interventions: a protocol for a scoping review. BMJ Open 2020;10:e039263. doi:10.1136/ bmjopen-2020-039263

- Prepublication history and additional material for this paper are available online. To view these files, please visit the journal online (http://dx.doi. org/10.1136/bmjopen-2020039263).

Received 13 April 2020

Revised 15 September 2020

Accepted 11 October 2020

Check for updates

(c) Author(s) (or their employer(s)) 2020. Re-use permitted under CC BY-NC. No commercial re-use. See rights and permissions. Published by BMJ.

Adelaide Health Technology Assessment (AHTA), School of Public Health, The University of Adelaide, Adelaide, South Australia, Australia

Correspondence to Dr Abdolvahab Baghbanian; abdolvahab.baghbanian@ adelaide.edu.au

\section{ABSTRACT}

Introduction In healthcare policy and economic literature, research on the health technology assessment (HTA) of complex interventions (Cls) is becoming increasingly important. In many developed countries, HTA guides decision-making to help achieve greater value for money when funding health care. However, research has yet to identify the forms of evidence and evaluation criteria that should be used in the HTA of Cls. Previous research has established that the HTA of Cls requires multiple factors to be evaluated but there is no agreement on which factors ought always to be considered. There is equally little agreement on which forms of evidence ought to be collected or synthesised and how. We plan to perform a systematic scoping review in order to identify the range of evaluation criteria and types of evidence currently used in the HTA of Cls.

Method and analysis This protocol was developed to guide the methodological framework for the conduct of a scoping review on health technology assessment (HTA) of complex interventions (Cls), using the Joanna Briggs Institute guidelines and the six-stage framework proposed by Arksey and 0'Malley, in addition to more recent innovations in scoping review methodology. A grey literature search will supplement the primary searches of seven electronic databases for studies available in English between January 2000 and August 2020. Two reviewers will independently screen all search results for inclusion and data will be extracted using a customised data extraction or charting form. Any dispute will be resolved by consensus or through arbitration by a third author. The mnemonic Population, Concept and Context will be adopted to establish criteria for selecting relevant literature, and the Preferred Reporting Items for Systematic Reviews and Meta-Analyses: Extension for Scoping Review will be used for reporting the results. Several explanatory-descriptive methods will be used for analysing the extracted data including frequency and trend analyses as well as reflexive thematic coding and analysis. Mapping evidence on the HTA of Cls will allow us to gain a better understanding of both established and emerging practices, including the information types, requirements, values and parameters that are incorporated in the HTA of Cls. We also expect the findings of the scoping review to help identify research gaps that will guide future studies. As healthcare becomes more complex in its delivery, it is timely to determine how these complex interventions should be assessed so that policy decisions can be made about whether implementation and public funding is warranted.

\section{Strengths and limitations of this study}

This protocol outlines the methodological framework for conducting a scoping review of health technology assessment methods to evaluate complex interventions.

- The scoping review aims to systematically search, identify and map different understandings of complex interventions. It also proposes to map and synthesise the approaches used in health technology assessment to evaluate complex interventions.

- The scoping review will be driven by an improved analytical method grounded in previous research.

- The review is limited to English-language studies published between January 2000 and August 2020.

- This review may not include all health technology assessment methodologies for complex interventions as some may not have been publicly documented.

Ethics and Dissemination This scoping review will involve secondary analysis of already collected data, and thus, does not require ethics approval. The research findings will be submitted to peer-reviewed journals for publication and will also be disseminated at conferences and seminars.

\section{INTRODUCTION}

Recent improvements in health outcomes for patients have largely been attributed to the development of innovative approaches to delivering healthcare as well as the increasing adoption, diffusion and use of health technologies or models of service delivery. ${ }^{1-3}$ However, previous research has emphasised that new and emerging health technologies or interventions are not always accessible, available or affordable to all potential patients. $^{4-9}$ This is particularly a problem when the medical condition is chronic and the intervention is complex, for example, caring for patients with dementia. ${ }^{10-13}$

Health technology assessment (HTA) was introduced in the 1980 s to guide the development of evidence-based health policy and funding decisions. ${ }^{12} 14$ It has already facilitated patient access to cost-effective 
technologies that improve health outcomes, and contributes to value for money investment in the context of scarce health resources. ${ }^{1516}$ Complex interventions (CIs) are being increasingly studied using HTA methods but clarification and guidance is needed on how CIs should be assessed to properly inform public funding decisions in healthcare.

The HTA of CIs is an emergent area of research ${ }^{17-19}$ yet it is somewhat complex and fragmented because it has not yet been systematically studied and there appears to be no consensus on what constitutes a CI. We are, therefore, proposing to conduct a scoping review of the approaches used by HTA practitioners when assessing CIs to help guide decisions on the funding of these interventions. This protocol is for such a systematic scoping review of the literature.

Health policy-makers and professionals increasingly insist on good evidence to support decisions about the development, adoption, acquisition, appropriateness, utilisation, pricing, procurement and reimbursement of health technologies. ${ }^{20}$ The development and expanding use of HTAs in the public and private sectors in developed economies and recently in low to middle-income countries $^{21-27}$ reflect this insistence. In particular, the study of the HTA of CIs has gained prominence in recent years. ${ }^{172829}$

According to the Australian Department of Health ${ }^{16}$ an HTA includes a variety of processes and mechanisms that use scientific evidence to evaluate the safety, efficacy, clinical effectiveness and cost-effectiveness of health services. Without concrete evidence, however, the uptake and diffusion of health technologies is likely to be influenced by a range of social, financial and institutional factors, resulting in suboptimal health outcomes and inefficient use of resources. ${ }^{30}$

The International HTA Definition Joint Task Group ${ }^{31}$ defined HTA as:

A multidisciplinary process that uses explicit methods to assess the value of a health technology at different points in its lifecycle. The purpose is to inform decision-making to promote an equitable, efficient, and high-quality health system.

In turn, a health technology comprises any intervention-being a test, device, medicine, vaccine, procedure, programme or system-developed to assess, prevent, diagnose or treat medical conditions; promote health; provide rehabilitation; organise healthcare delivery; or support physical, psychological or social functional and behavioural changes. ${ }^{32}{ }^{33}$ A health intervention has also been broadly defined as 'an act performed for, with or on behalf of a person or population whose purpose is to assess, improve, maintain, promote or modify health, functioning or health conditions'. ${ }^{32}$

By contrast, there is no agreed definition of a ' $\mathrm{CI}$ ' in health. A broad, inclusive definition of a CI includes the following components. ${ }^{34-38}$
1. A CI contains multiple elements or components that adapt to systemic and environmental changes (there is intervention complexity).

2. A CI has simple, complicated or multiple causal pathways with feedback loops, and it has mediators and moderators with synergistic effects (there is pathway complexity).

3. A CI emphasises non-linear relationships, interactions and interconnections between components, for example, communication and control (there is interaction complexity).

4. A CI targets multiple actors, for example, individuals, groups and/or institutional levels (there is population complexity).

5. A CI is responsive or sensitive to initial conditions. Changes in initial conditions may present emerging behaviour and variable responses or outcomes whose details are not predictable (commonly referred to as 'the butterfly effect').

6. A CI requires multifaceted adoption, uptake or integration methods, perspectives or strategies (there is implementation complexity).

7. A CI exists within systems and operates in a dynamic multidimensional environment (there is contextual complexity).

8. A CI causes practical and methodological difficulties for assessors (there is evaluation complexity).

In a nutshell, a CI is a heath technology with multiple components that interact synergistically in non-linear, dynamic pathways that depend on their history (initial conditions) and a context in which patterns, reactions and outcomes are not easily predicted.

Interventions can be understood as being simple, complicated, complex or chaotic. Formal rule-based interventions with anticipated impacts fall into the simple (known) domain. An example of a simple intervention can be vaccination, medication or injection of insulin in accordance with protocols in place. In this domain, clinical practitioners and patients can easily standardise the procedure and it is known what the effects and consequences of the mechanisms are.$^{39} 40$ Complicated interventions correspond to the knowable domain, meaning that the relationship between cause and effect may not be fully known by all decision-makers but can be known through reductionist ways of thinking and objectivity, meaning that human behaviour or complex phenomenon can only be explained by breaking it down into smaller/ simpler component parts. ${ }^{41}$ An example of a complicated intervention could be a surgical operation where expertise, detailed protocol and latest scientific evidence are all required in order to do the procedure. ${ }^{1840} 42$ Simple interventions call for 'best practices' and complicated ones apply 'good practices'. ${ }^{43}$

CIs, on the other hand, sit in the domain of 'emergent practice' that cannot be solved with 'best' or 'good' practices alone. For CIs, a fixed or static evaluation is unlikely to be sufficient; rather, experimentation is required to probe the components of the intervention and determine 
which factors are critical to its success. In this domain, the relationship between cause and effect can only be perceived in retrospect, not in advance. ${ }^{44}{ }^{45}$ Integrated care, behavioural interventions for medication compliance and outpatient palliative care services are examples of CIs, where the success in managing the problems of the first patient is not an indication that others will be treated effectively since no fixed rules, guidelines or formulae exist to warrant success. ${ }^{37} 394647$ Cognitive-behavioural therapy (CBT) for depression, for instance, consists of multiple components that interact to improve the condition, even though we may not be sure how these components interact, nor what they do to each other. Such an intervention may involve multiple treatment approaches, aimed at providing support on multiple levels (eg, individuals, groups, systems and community) by various professionals and different means and in a non-linear manner. Changes in results are not proportional to any changes in input, and the technique may require multifaceted training or adoption, and require special consideration of the environment or the context in which CBT is developed, implemented and assessed.

HTA methods have been developed to assess the quality, safety, efficacy, effectiveness and cost-effectiveness of health interventions and programmes, but CIs are often complex in ways that pose challenges to the use of conventional HTA methods. Current HTA methods and practices have largely been tailored towards simple and complicated interventions where specific plans, procedures, techniques, guidelines and protocols are developed to direct action and achieve outcomes. HTA traditionally occurred at the point of a new technology's entry into the health system, rather than once the technology was established. HTAs to inform a public funding decision were uncommon when it related to changes in the organisation and delivery of established healthcare. With recent moves in some health systems to integrate HTA practices into implementation science and health technology management, ${ }^{28} 4849$ the types of interventions being evaluated have changed. While HTA methods work quite well in simple and complicated situations, there is a growing need to improve how HTA handles CIs in practice.

Previous research has recognised that HTA is multifactorial. Healthcare decision-makers rely heavily on HTAs to achieve greater value for money, an overarching concept that encompasses comparative safety, relative effectiveness, cost-effectiveness, social consequences, organisational implications and legal and ethical aspects of a technology. ${ }^{50-53}$ However, there is a view that it does not fully consider the real-world context, complexities and contingencies relating to health technologies, and relies more on isolating single factors. ${ }^{18} 4454$ Recent studies on HTAs of CIs have identified many relevant factors such as context (eg, setting and socioeconomic or sociocultural aspects), implementation issues, patientrelated factors and preferences, safety profile and innovation level. ${ }^{55}{ }^{56}$ However, the relevance of these studies has been constrained by the applicability of HTA reports on CIs across different jurisdictions and various levels of government or decision making. ${ }^{57}$ Recognition that CIs are contingent on their context not only has implications for the effect of the CIs but also for their acceptability; feasibility of development, evaluation and implementation; and sustainability. ${ }^{58}$

There is no apparent consensus on what constitutes a CI and how different stakeholders, experts or researchers define it, nor what evidence and evaluation criteria should be used in the evaluation of CIs (and hence HTA-based decision making). Thereby, it is unclear what dimensions of context foremost determine if a technology should be funded or not. It is also unclear how HTA is being conducted at the international level to formally evaluate CIs and to what extent the proposed or used evaluation criteria and types of evidence differ according to the definitions used.

In view of these research gaps, we will conduct a systematic scoping review to map how CIs are defined in HTA and also determine the range of methods used to evaluate CIs in HTA. This will help to identify any knowledge gaps. More importantly, it will help HTA experts and health policy-makers to understand the types of information, criteria, requirements and processes that currently feature in HTAs of CIs, and thus better equip them to decide between options for conducting such HTAs.

We describe the protocol proposed for undertaking and reporting this scoping review.

\section{METHODS AND ANALYSIS}

This scoping review protocol was designed to guide the formulation of review aim(s) and questions, eligibility criteria and conduct characteristics of the review.

Previous studies have proposed a variety of review types to synthesise the available evidence, of which the 'scoping review' or 'scoping study' approach has recently gained considerable popularity and recognition as a transparent and systematic methodology for examining a broadly covered but unclear topic. The aim is to provide a comprehensive map of the available literature and to identify key concepts, types of evidence, theories or research gaps. ${ }^{59}$ This allows research scholars and policy-makers to explain the working concepts and theoretical limits of a particular topic, and to make informed decisions within a shorter time frame. ${ }^{6061}$ The first scoping review framework was most likely published in $2005,{ }^{62}$ and as such, this form of analysis is a relatively new approach ${ }^{63}$ for which there is still no universal definition, consistent application or definitive procedure for performing or reporting in all research fields. ${ }^{6364}$ The following features, however, are specific to most definitions:

Scoping reviews (or scoping studies), also known as 'mapping' reviews, represent a method of evidence synthesis to explore research concerns, ${ }^{61}{ }^{65}$ identify research gaps, ${ }^{62}$ update empirical evidence on new research areas, ${ }^{65}$ summarise and disseminate findings 
from a body of knowledge that is heterogeneous in methodology or discipline, ${ }^{66}$ map or chart the extent (ie, volume, range and type) as well as the nature and characteristics of evidence underpinning a research area, ${ }^{6267}$ and may be used where the research area is broad, complicated or not comprehensively reviewed.$^{6167}$

Scoping reviews are not necessarily a quick alternative to systematic reviews. ${ }^{62}$ They represent a type of synthesis rather than a type of systematic review, but many of the steps and processes taken in systematic reviews are reflected in scoping reviews. The differences are slight and relate to the research aims and aspects of method. Scoping reviews, for instance, typically do not include critical appraisal, nor are the findings used to produce policy or practice recommendations since they are reviews that describe rather than evaluate and report. ${ }^{60}$

This scoping review will be part of a large-scale study on the 'HTA of CIs'.

\section{The rationale for the choice of scoping review methodology}

For this study, we determined that a scoping review would be the most appropriate method of collating the research evidence on how HTA is undertaken for CIs. The HTA of CIs is an emerging research area that is still fragmented, complex, broad, poorly understood, understudied or not researched in detail. ${ }^{68} 69$ The scoping review represents the most suitable method for responding to the questions of the proposed study, as it aims to outline, map, synthesise and disseminate different concepts and types of evidence that exist around the conduct of HTA in relation to CIs, and identify the gaps for further research.

Scoping reviews are susceptible to bias and must be rigorously and transparently designed and prepared to ensure the findings and reports are accurate and relevant for end users. ${ }^{616265667071}$ The (The Preferred Reporting Items for Systematic Reviews and Meta-Analyses Extension for Scoping Reviews (PRISMA-ScR) ${ }^{66}$ will form the basis for reporting findings in the present scoping review. The PRISMA-ScR checklist is mainly used to ensure the findings are adequately reported and that the review is replicable and therefore credible. ${ }^{72}$

\section{Registration of the review protocol}

In line with systematic review methodology ${ }^{73}$ and scoping review methodology, ${ }^{74}$ the current study begins with a protocol developed before undertaking the actual scoping review in order to minimise bias in the review process. Developing such a protocol is critical as it predefines the research aim(s), questions and methods to be addressed, with criteria for the inclusion and exclusion of literature that relates to the scoping review's aim(s) and question $(\mathrm{s})$. The protocol provides a systematic approach to the conduct and reporting of the review, allows for transparency of the process, and enables readers to understand how the findings of the scoping review have been arrived at. Unlike a typical systematic review, which seeks to answer a specific or series of question(s) - based on very precise inclusion criteria, for example, on the basis of the Population, Intervention, Comparator and Outcome (PICO) elements—-the scoping review has a wider 'scope' with correspondingly less restrictive inclusion criteria. It is intended to respond to a series of broad/open questions based on the elements of the (Population, Concept and Context (PCC) inclusion criteria. ${ }^{75}$ Since the purpose of the proposed scoping review is to identify, explore and map international efforts related to the conduct of HTA of CI-including the processes and/or methodologies used in the HTAs of CIs-we used the latter.

This protocol was prospectively registered with the Open Science Framework (https://osf.io/kv9hu/) since the PROSPERO database does not accept systematic scoping review protocols. An official record of this protocol promotes transparency and assists in reducing duplication of the work, as will this protocol publication.

\section{Patient and public involvement \\ No patient involved.}

\section{Stages of the proposed scoping review}

The scoping review outlined in this protocol will be driven by an improved analytical method grounded in previous research. This will conform to Arksey and O'Malley's sixstage methodological framework ${ }^{62}$ but feature further refinements made by Levac, $e t a l^{61}$ and the Joanna Briggs Institute (JBI) ${ }^{75}$ We will also draw on insights from more recent innovations in Arksey and O'Malley's framework made by Colquhoun $e t a l^{65}$ and Tricco $e t a b^{66}$ in setting out the plan and reporting of the review.

We will rely on the following six stages in this scoping review: (1) developing the research question, (2) identifying relevant studies, (3) selection of eligible studies, with the establishment of a set of inclusion and exclusion criteria; (4) charting the data, that is, sifting, charting and sorting information based on the key issues and themes; (5) collating, summarising and reporting the results, and (6) consulting key stakeholders to inform and validate study findings.

\section{Stage 1: developing the research question(s)}

The first stage in undertaking a scoping review is research question formulation and linking the question(s) to the study aims/objectives. ${ }^{61}{ }^{62}$ Research questions will direct the subsequent stages of this review. Whereas systematic reviews address specific and focused research questions, ${ }^{73}$ scoping studies feature research questions that are broad and the focus is on mapping and summarising the extent and nature of the evidence. ${ }^{6165}$

The main research question for this scoping review is 'how is HTA being done by HTA evaluation agencies to formally evaluate CIs at the international level?'. The research subquestions are:

- What different definitions of a CI exist in the literature, and how do these definitions differ?

- What evaluation criteria and domains are assessed in the HTA of CIs? 
- To what extent do the evaluation criteria and evidence types differ according to the CI definitions used?

- Does the approach differ from country to country?

- What is current practice in the HTA of CIs in Australia? We have a particular interest in what is being done to evaluate CIs in Australia, so we are prespecifying Australia for subgroup analysis as part of our review.

The proposed scoping review will use the PCC framework to align the study selection with the research question. The $\mathrm{JBI}^{75}$ recommends PCC as a less restrictive alternative to the PICO (recommended for systematic reviews) for the construction and interpretation of scoping review questions and to describe elements of the inclusion criteria.

\section{Stage 2: identifying relevant studies}

The identification of relevant studies and information sources involves the creation of a search strategy, underpinned by inclusion and exclusion criteria. These criteria are categorised under the broad PCC mnemonic recommended for scoping reviews, as set out below. ${ }^{75}$ We sought the advice of a senior medical librarian to apply the search strategy to the widest possible degree, since the main attribute of a scoping review is full coverage of the literature on the subject. As a consequence, the search strategy will follow the three-phased JBI process, ${ }^{75}$ and extend to three more additional phases as follows:

The first phase entails the identification of indexing terms and text words. To inform these, a scoping search of two relevant online databases-that is, Medline (PubMed) and Embase-will be pilot tested to compile and preselect a list of keywords from titles, abstracts and index terms used in publications most relevant to the topic. Citations will be downloaded into an Endnote database and keywords and indexing terms will be identified. Use of the PubReMiner (a free version data mining tool: http://hgserver2.amc.nl/cgi-bin/miner/miner2.cgi) will also retrieve the most frequently occurring keywords and indexing terms (Medical Subject Headings, MeSH) that will be incorporated into the final search query. The keywords and Mesh terms retrieved will provide the foundation for creating a definitive search strategy through Medline (PubMed interface). Using an adapted version of the PRESS Evidence-Based Checklist for Peer Review of Electronic Search Strategies, this preliminary search strategy will be peer-reviewed independently by external HTA evaluators with information specialist expertise ${ }^{76}$ and readjusted following the feedback.

The review will focus on English language evidence published worldwide between January 2000 and August 2020, and also a purposive sample of selected countries (and their respective HTA bodies) for their HTA reports and guidance documents. This consists of a diverse mix of countries including members of International Network of Agencies for Health Technology Assessment (INAHTA) ( www.inahta.org) as indicated in box 1 .

In choosing target countries, the review aims to include countries that have a diverse set of HTA mechanisms in
Box 1 Selected countries (and their respective HTA bodies)

INAHTA Members

ACE-Agency for Care Effectiveness, Singapore

AETS-Agencia de Evaluación de Tecnologias Sanitarias, Spain

AETSA-Andalusian Agency for Health Technology Assessment, Spain

Agenas-The Agency for Regional Healthcare, Italy

AHRQ-Agency for Healthcare Research and Quality, United States of America

AHTA-Adelaide Health Technology Assessment, Australia

AlHTA -Austrian Institute for Health Technology Assessment, Austria

AOTMiT-Agency for Health Technology Assessment and Tariff System, Poland

AQuAS-Agència de Qualitat i Avaluació Sanitàries de Catalunya, Spain ASERNIP-S-Australian Safety and Efficacy Register of New Interventional Procedures -Surgical, Australia

ASSR-Agenzia Sanitaria e Sociale Regionale (Regional Agency for Health and Social Care), Italy

AVALIA-T-Galician Agency for Health Technology Assessment, Spain

CADTH-Canadian Agency for Drugs and Technologies in Health, Canada

CDE -Center for Drug Evaluation, Taiwan, Republic Of China

CEDIT-Comité d'Evaluation et de Diffusion des Innovations Technologiques, France

CHQAC-Center for Healthcare Quality Assessment and Control, Russian Federation

CONITEC-National Committee for Technology Incorporation, Brazil

DEFACTUM-Social \& Health Services and Labour Market, Denmark

FinCCHTA-Finnish Coordinating Center for Health Technology Assessment, Finland

G-BA-The Federal Joint Committee (Gemeinsamer Bundesausschuss), Germany

G0eG-Gesunheit Österreich, Austria

HAD-Uruguay-Health Assessment Division, Ministry of Public Health, Uruguay

HAS-Haute Autorité de Santé, France

HTRG-Health Technology Reference Group, Australia \& NEW Zealand

HIQA-Health Information and Quality Authority, Ireland

HIS-Healthcare Improvement Scotland, United Kingdom

HQO-Evidence Development and Standards Branch, Canada

HTW-Health Technology Wales, United Kingdom

IACS-Health Sciences Institute in Aragon, Spain

IECS-Institute for Clinical Effectiveness and Health Policy, Argentina

IETS-Instituto de Evaluación Tecnológica en Salud, Colombia

IETSI-Institute of Health Technology Assessment and Research, Peru

IHE-Institute of Health Economics, Canada

INEAS-National Authority for Assessment and Accreditation in Healthcare, Tunisia

INESSS-Institut national d'excellence en santé et en services, Canada IQWiG-Institut für Qualität und Wirtschaftlichkeit im Gesundheitswesen, Germany

KCE-Belgian Health Care Knowledge Centre, Belgium

MaHTAS-Health Technology Assessment Section, Ministry of Health Malaysia, Malaysia

NECA-National Evidence-based healthcare Collaborating Agency, Korea

NICE- National Institute for Health and Care Excellence, UK

NIHR-National Institute for Health Research, UK

NIPH-Norwegian Institute of Public Health, Norway

OSTEBA-Basque Office for Health Technology Assessment, Spain

RCHD-Ministry of Public Health of the Republic of Kazakhstan, Republican Centre for Health Development, Kazakhstan 


\section{Box 1 Continued}

SBU-Swedish Agency for Health Technology Assessment and Assessment of Social Services, Sweden

SEC-Department of HTA at the State Expert Centre of the Ministry of Health, Ukraine

SFOPH-Swiss Federal Office of Public Health, Switzerland

UVT-HTA Unit in A. Gemelli Teaching Hospital, Italy

ZIN-Zorginstituut Nederland, The Netherlands

ZonMw-The Netherlands Organisation for Health Research and Development, The Netherlands

\section{Non-INAHTA Members}

The Department of Health Technology Assessment, Iran

HIRA-Health Insurance Review and Assessment Service) South Korea

Institute of Healthcare Technology Assessment, Shomachi and

Department of Technology Assessment and Biostatistics, Japan

HiTAP-Health Intervention and Technology Assessment Program,

Thailand

CMTP-Centre for Medical Technology Policy, United States of America

KDTD Turkish Evidence Based Medicine Association, Turkey

CMeRC-HTA Unit Charlotte Maxeke Research Cluster-Health technology

Assessment Unit, South Africa

place (eg, clinical benefit assessment plus economic evaluation verses clinical benefit assessment alone) along with those with different levels of economic development from high-income, middle-income and low-income countries. The rationale for selection of these counties would also be the variation in their health system financing (eg, tax-supported, social security-based or private insurance corporations and out-of-pocket), the organisation of the healthcare delivery system (centralised verses decentralised structure), and the perspective used in HTA (health system verses societal perspective), so that the sample can be representative of the major types of health systems and HTA approaches across different nations.

The rational for choosing the INAHTA HTA agencies is that these agencies

- Assess technology in healthcare.

- Are non-profit organisations.

- Relate to a regional or national government.

- Are funded at least $50 \%$ by public sources.

- Provide free access to publicly available reports to other INAHTA members on request. (www.inahta. org).

Data are limited to evidence published in English language because the research team is unable to obtain and translate non-English literature and remain within the expected project timeline. However, we will retain the abstracts/summary for non-English studies with English abstracts that seem to correspond to our inclusion criteria (aside from the language) for the purposes of reporting.

Keywords and MeSH terms selected for the scoping search (based on the PubMed platform) include: (("complex intervention"[tiab] OR "complex technology"[tiab] OR “complex technologies"[tiab] OR "complex program"[tiab] OR "complex programme" [tiab] OR "multi-component intervention"[tiab] OR "multi-component program"[tiab] OR "multi-component programme"[tiab] OR "multi-part intervention"[tiab] OR "multi-part program" [tiab] OR "multi-part programme"[tiab] OR "multifactorial intervention" [tiab] OR "bundled intervention"[tiab] OR "bundled program" [tiab] OR "bundled programme" [tiab]) AND ((HTA OR "health technology assessment" OR "Technology Assessment, Biomedical" [Mesh] OR "Comparative Effectiveness Research"[Mesh] OR "Evidence-Based Medicine”[Mesh] OR evaluat*[tiab] OR assess*[tiab] OR reimburs*[tiab] OR fund*[tiab] OR insur*[tiab] OR "health technology management") OR ("Health"[Mesh] AND ("Decision Making"[Mesh] OR "Decision-mak*"[tiab] OR "value assessment" OR Technology Assessment [tiab] OR policy [tiab] OR "Policy Making" [MAJR]))). The yield from the electronic database search will be recorded in a table.

The second phase includes developing and implementing unique database queries for each database included in the protocol, based on the list of keywords and index terms retrieved from the previous phase. Apart from Medline/PubMed, these databases will include the Cochrane Library [Cochrane Database of Systematic Reviews and Cochrane Central Register of Controlled Trials ], PsycINFO, CINAHL, Embase, Epistemonikos and the INAHTA HTA database.

The third step builds on the data analysis of the preliminary searches and involves a manual search for potential publications not found through the online search of databases by reviewing the archives of three leading journals with the highest number of published papers in HTA of CIs.

In the fourth phase, a search will be performed to identify grey literature on the topic. This requires manual searches of any non-indexed and unpublished literature of relevance to this review including research in progress, theses, in-press articles, technical HTA reports, guidelines and procedure documentation concerning the evaluation of CIs. Searches will be conducted through Google, websites of HTA international (https:/ / www.htai. org/), the International Society of Pharmacoeconomic Outcomes Research (https://www.ispor.org/), as well as and the websites of HTA agencies that are members of INAHTA (http://www.inahta.org/) ${ }^{77}$ The HTA websites and repositories outlined above will be reviewed for guidance documents and actual reports of HTAs of CIs. The search strategy in this phase will help avoid publication bias and identify any other relevant material(s) for inclusion, so the study will not be narrowed to only peerreviewed papers, noting that HTA is a policy science and much of the documentation does not make it into peer reviewed journals. If necessary, authors of original guidance documents and HTA reports will be contacted for further information or missing data.

On the successful completion of Stage 2 that is, the search strategy, search results (final studies identified) will be collated and imported into the most recent EndNote software (Clarivate Analytics), with duplicate entries removed. 
Table 1 PCC framework for selection of eligible studies

\begin{tabular}{|c|c|c|}
\hline PCC element & $\begin{array}{l}\text { Definition/determinants: (per JBI reviewer's } \\
\text { Manual Ch.11) }\end{array}$ & Inclusion criteria \\
\hline $\begin{array}{l}\text { P-Population* } \\
\text { (Who?) }\end{array}$ & $\begin{array}{l}\text { Documents produced by HTA agencies } \\
\text { or HTA networks, HTA evaluators or HTA } \\
\text { methodologists }\end{array}$ & $\begin{array}{l}\text { Published studies and grey literature in the form of HTA } \\
\text { reports or HTA guidance or methods documents } \\
\text { HTA reports include secondary research studies that } \\
\text { involve systematic reviews, realist reviews, meta- } \\
\text { analyses, meta-syntheses, mixed-methods reviews, } \\
\text { qualitative reviews, rapid reviews with/without } \\
\text { economic evaluations, budget impact analyses and } \\
\text { ethical, social, legal and organisational analyses } \\
\text { undertaken to specifically inform a health policy } \\
\text { decision. } \\
\text { Studies showing that their full-texts are available } \\
\text { in English through academic journals, institutional } \\
\text { repositories, archives or other collections of scientific } \\
\text { and other articles }\end{array}$ \\
\hline $\begin{array}{l}\text { C-Context } \\
\text { (With What } \\
\text { Qualifiers?) }\end{array}$ & $\begin{array}{l}\text { All settings are considered. } \\
\text { HTA must be conducted for an access or } \\
\text { funding decision whether at the national, } \\
\text { regional or hospital level. }\end{array}$ & $\begin{array}{l}\text { Global search for all published studies and grey } \\
\text { literature } \\
\text { Search of a purposive sample of selected countries } \\
\text { (and their respective HTA bodies) for their HTA reports } \\
\text { and guidance documents from the INAHTA member list } \\
\text { (www.inahta.org) } \\
\text { English language studies available between January } \\
2000 \text { and August } 2020\end{array}$ \\
\hline
\end{tabular}

*Population/participant entails important characteristics of the study population, setting or participants such as age, gender and other qualifying criteria.

†Concept includes details that relate to elements that would be detailed in the scoping review such as the 'Cls', HTA, outcomes or other 'phenomena of interest'. The concept should be clearly articulated to guide the scope and breadth of the research.

$\ddagger$ Context is defined as the conditions and circumstances that are relevant to the application of an intervention, for example, setting (eg, hospital) and sociocultural aspects (knowledge, beliefs, conceptions, customs, institutions and any other capabilities and habits acquired by a group that may influence uptake). ${ }^{56}$ Cultural factors, socioeconomic issues, geographical location, politics, specific racial preferences, gender interests may be included in context.

$\mathrm{Cl}$, complex intervention; HTA, health technology assessment; INAHTA, International Network of Agencies for Health Technology Assessment; JBI, Joanna Briggs Institute.

$\mathrm{AB}$ will develop the searches and these will be peer reviewed by TM and independently by an information specialist. On finalisation of the search strategy, $\mathrm{AB}$ will conduct the electronic searches. The search strategy will be limited to the English language, and those studies published between January 2000 and August 2020. Year 2000 was chosen as the starting point for this review as many HTA and CI guidelines were developed from this date. ${ }^{78} 79$ The search will be updated just prior to submitting the results for publication.

\section{Stage 3: screening and selection process (selection of eligible studies)}

Eligibility criteria

The relevant documents to address the research question will be selected using the PCG study selection criteria (table 1).
The screening and selection of eligible studies will be carried out in two phases, beginning with the initial screening for relevant studies based on the title and/or abstract, followed by full-text review of those studies selected at the screening stage. Both phases will involve independent duplicate screening of a random $10 \%$ of the records to ensure the screening approach taken is reliable. A kappa score will be calculated to determine inter-rater reliability. Reviewers will confer to determine the reason for any differences in study selection and the approach will be amended accordingly. Any paper over which there is a lack of consensus for inclusion in the review will be adjudicated by a third independent reviewer. ${ }^{6380}$ A single reviewer will screen the remaining database.

In the first screening stage, the citation or study record will be screened as 'included, excluded or uncertain'. In the 
second stage, the full-text article will be retrieved of those categorised as included or uncertain and the study selection criteria applied again until there is a definitive decision regarding study inclusion. The third screening stage consists of checking the reference lists of included studies-that is, all included studies will be scanned and cross-checked for more relevant studies (backward citation searching).

We will document the reasons for the inclusion of uncertain studies and exclusion of other resources in a PRISMA-ScR flow diagram. ${ }^{66}$ Details of the literature screening and results obtained will be outlined narratively and presented in a graphical diagram developed from the PRISMA-ScR flow chart ${ }^{66}$ or in accordance with the JBI guideline. ${ }^{75}$

\section{Exclusion criteria}

We will exclude studies that specifically do not meet the above inclusion criteria. Studies without evidence of HTA of CIs, those conducted or published in languages other than English and those where the full-text cannot be accessed will be excluded. Exclusions will also apply to other forms of publications including letters, commentaries or editorials, narrative reviews, study protocols, as well as conference abstracts and presentations.

\section{Stage 4: extracting and charting the data}

In this stage, the data from all the studies included will be collected and charted using an adapted or structured descriptive-analytical approach. The process by which data is extracted in scoping reviews is termed 'charting' where a logical and descriptive summary of the study results must be recorded corresponding to the aims and questions of the scoping review. ${ }^{63} 7282$ The data extraction template (known as data charting form or data abstraction tool) will reflect all the variables that are consistent with the research questions and aims of the scoping review. ${ }^{63}{ }^{83}$ It is designed in detail to capture the relevant information about the study's key characteristics and detailed information about all the terms and metrics used to describe and summarise the phenomena of interest, that is, the HTA of CIs. The supplementary file contains the 'data extraction and charting form' and outlines a detailed list of these components (online supplemental table 1). The data extraction form presented in the supplementary file will be piloted with not more than $10 \%$ of the included studies by the key reviewer $(\mathrm{AB})$, and further examined within the research team to ensure accuracy and consistency of the extracted data. ${ }^{84}$

\section{Stage 5: collating, summarising and reporting the findings}

The distinctive purpose of conducting a scoping review is to compile the results and to provide an overview of narrowly defined matters rather than a meta-synthesis of results. ${ }^{62}$ This stage aims to summarise the findings collected in order to explore the known and unknown aspects of the given topic and to identify areas that need to be studied further. ${ }^{82} 83$

Stage 5 will primarily draw on recent innovations in reporting scoping review results including the PRISMA-ScR. ${ }^{66}$ It will also make use of an interpretative methodology for presenting a narrative or descriptive account. A narrative synthesis will interpret and summarise the extracted findings in relation to the proposed scoping review question and in the light of the main research aims. ${ }^{84-86}$ Some researchers argue that the inherent challenges to undertaking a scoping review is to establish a framework for presenting the narrative account. ${ }^{8285}$ This will be achieved through an inductive data analysis approach such as reflexive thematic analysis. ${ }^{87} \mathrm{In}$ doing so, we will import the extracted data from the charting tool into the NVivo software V.12 ${ }^{88}$ for coding and analysis. Themes will then be created and their relationship to the research question and aims will be examined. The collection, abstraction and reporting process will also draw on previous works, where a visual representation or numerical overview of amount, distribution and evolution (ie, frequency and trend analyses), and nature of evidence on HTA of CIs will be presented through figures or tabular formats. ${ }^{61} 728689$ The mapping approach suggested by Miake-Lye et al will be specifically used to enhance the mapping results from the literature screening stage. ${ }^{90}$ The process of mapping the qualitative data, if any, will follow an initial coding, focused coding (categorisation), data displaying through diagrams or tables, pattern and themes identification, summarising and synthesising. Such 'evidence mapping' is pivotal to performing a scoping review and displays the research context or environment graphically. These proof maps provide better identification of research patterns and themes and help to postulate explanations for summarising and synthesising the results.

Finally, the meanings and implications of the findings in relation to the aims of the study will be discussed and reported for future research, policy, and practice.

\section{Methodological quality appraisal}

Competing arguments exist regarding whether scoping reviews ought to include the methodological quality appraisal of primary studies. While some support inclusion, many others object. According to Peters et $a l^{63}$ a formal quality assessment of the primary studies is not generally performed within a scoping review because unlike systematic reviews, scoping reviews are mainly designed to provide an overview or map of the existing evidence base regardless of quality. As this research is aimed at mapping the available research ${ }^{64}$ on HTAs of CIs, this scoping review will not undertake any formal assessment of the methodological quality of the included studies.

\section{Stage 6: consulting key stakeholders}

The present scoping review constitutes the first study of a five-stage research project. There will not be any consultation exercise or stakeholder involvement during the scoping review. However, stakeholder consultation will occur in the later stages of the major research project. A web-based survey and a two-round Delphi study of decision-makers, HTA experts and other stakeholders will be conducted to obtain more information and perspectives regarding the definition of CI and the HTA methods for evaluating CIs. ${ }^{62}$ 


\section{DISCUSSION}

HTA has been developed as a form of policy science that aims to inform policy and practice in the delivery and financing of healthcare interventions and technologies. ${ }^{91}$ New health technology, however, is not always available to all potential patients, accessible, or affordable. ${ }^{6-8}$ This is a problem for CIs. ${ }^{10} 11$ Despite many advances in the application, implementation, methods and practices of HTA in Australia and other international settings, there remains a need to improve the transparency and consistency in regulatory and reimbursement decisions related to CIs. The HTA of CIs is an emergent area of research yet understanding of it is somewhat complex and fragmented because it has not yet been systematically studied.

To the best of our knowledge, there is no comprehensive scoping review of the HTA of CIs. Mapping evidence on the HTA of CIs will enable us to better understand both established and emerging practices, including the information types, criteria and values that are used in the assessment of these interventions. We developed an a priori protocol to undertake a systematic scoping review study on the HTA of CI across the world. The scoping review methodology proposed by Arksey and O'Malley ${ }^{62}$ will primarily guide the review, along with insights representing more recent innovations in the field. ${ }^{6165667585}$

We propose to adopt a step-by-step procedure that will adequately address the requirements for our scoping review, especially in terms of data extraction, data analysis and reporting of the results. This helps us to boost our approach's feasibility, rigour, reproducibility, reliability and credibility, including by minimising reporting bias. ${ }^{92}$

The review, however, is subject to a number of limitations. The main limitation of the current scoping review is that some HTA practices may remain undocumented or not publicly accessible, so even searching HTA agencies' websites may not provide the full picture of evaluation criteria used to assess CIs. The review is also limited in that we can only include evidence that is in English because of resource constraints. This is not an uncommon reason and may lead to some relevant research being excluded, ${ }^{93}$ although in the majority of cases the exclusion of non-English studies will have minimal impact on the overall conclusions of a review. ${ }^{94}$

We estimate the timeline for completion of this study to be 6 months. Any protocol amendments and non-compliance will be documented and reported transparently in the actual scoping review.

\section{Ethics and dissemination}

This scoping review will involve secondary analysis of already collected data and thus does not require ethics approval. The research findings will be submitted to peer-reviewed journals for publication and will also be disseminated at conferences and seminars.

Contributors All authors have made substantial intellectual contributions to the development of this protocol. $A B$ conceptualised the study and prepared the manuscript under the guidance and supervision of TM, DC and SW. All authors jointly conceived the idea for the project contributed to the study design and development of research questions. TM contributed to the methodology and critically reviewed the manuscript. DC provided detailed comments on earlier drafts. AB completed the final draft, and all authors read and approved the final manuscript.

Funding The authors have not declared a specific grant for this research from any funding agency in the public, commercial or not-for-profit sectors.

Competing interests None declared.

Patient consent for publication Not required.

Provenance and peer review Not commissioned; externally peer reviewed.

Supplemental material This content has been supplied by the author(s). It has not been vetted by BMJ Publishing Group Limited (BMJ) and may not have been peer-reviewed. Any opinions or recommendations discussed are solely those of the author(s) and are not endorsed by BMJ. BMJ disclaims all liability and responsibility arising from any reliance placed on the content. Where the content includes any translated material, BMJ does not warrant the accuracy and reliability of the translations (including but not limited to local regulations, clinical guidelines, terminology, drug names and drug dosages), and is not responsible for any error and/or omissions arising from translation and adaptation or otherwise.

Open access This is an open access article distributed in accordance with the Creative Commons Attribution Non Commercial (CC BY-NC 4.0) license, which permits others to distribute, remix, adapt, build upon this work non-commercially, and license their derivative works on different terms, provided the original work is properly cited, appropriate credit is given, any changes made indicated, and the use is non-commercial. See: http://creativecommons.org/licenses/by-nc/4.0/.

ORCID iD

Abdolvahab Baghbanian http://orcid.org/0000-0003-3651-337X

\section{REFERENCES}

1 Goodman CS. HTA 101: introduction to health technology assessment. MD: NAtional Library of Medicine (US),, 2014.

2 Godman B, Bucsics A, Vella Bonanno P, et al. Barriers for access to new medicines: searching for the balance between rising costs and limited budgets. Front Public Health 2018;6:328.

3 Godman B, Basu D, Pillay Y, et al. Review of ongoing activities and challenges to improve the care of patients with type 2 diabetes across Africa and the implications for the future. Front Pharmacol 2020;11:108.

4 OECD. Geographic variations in health care: what do we know and what can be done to improve health system performance? Focus on Health 2014.

5 WHO. Engagement and participation for health equity. reducing health inequities: perspectives for policy-makers and planners the regional office for Europe, world Health organization 2017:20.

6 Kastner M, Hayden L, Wong G, et al. Underlying mechanisms of complex interventions addressing the care of older adults with multimorbidity: a realist review. BMJ Open 2019;9:e025009.

7 Focus on Accessibility: Multimodal Healthcare Technology for All. The 2016 ACM workshop on multimedia for personal health and health care. Amsterdam, The Netherlands: ACM, 2016.

8 World Health Organisation. Universal health coverage: supporting country needs. Geneva: World Health Organization, 2013.

9 Baumgart DC, Misery L, Naeyaert S, et al. Biological therapies in immune-mediated inflammatory diseases: can biosimilars reduce access inequities? Front Pharmacol 2019;10:279.

10 Culo S. Risk assessment and intervention for vulnerable older adults. The BC Medical Journal 2011;53:421-5.

11 Khanassov V, Pluye P, Descoteaux S, et al. Organizational interventions improving access to community-based primary health care for vulnerable populations: a scoping review. Int J Equity Health 2016;15:168.

12 Booth A, Noyes J, Flemming K, et al. Guidance on choosing qualitative evidence synthesis methods for use in health technology assessments of complex interventions 2016.

13 Parker GM, Gridley K, Birks YF, et al. Using a systematic review to uncover theory and outcomes for a complex intervention in health and social care. Life Story Work for people with dementia as a worked example 2020.

14 Salisbury N, Castro H, Bump J. The emergence of health technology assessment organizations: lessons from five countries. Seattle, WA, USA: Program for Assessment of Technology in Health (PATH), 2017.

15 Busse R, Blümel M, Scheller-Kreinsen D, et al. Managing chronic disease in Europe. The Initiative for Sustainable Healthcare financing 
in Europe (2nd report) Securing Europe's healthcare future: Chronic disease management and health technology assessment. Prague: Berlin University of Technology, 2009.

16 Australian Department of Health. About health technology assessment: what is a health technology assessment Canberra, Australia: department of health, Australian government, 2019. Available: https://www1.health.gov.au/internet/hta/publishing.nsf/ Content/home-1 [Accessed 09 February 2020].

17 Rehfuess EA, Gerhardus A, INTEGRATE-HTA. INTEGRATE-HTA: adopting and implementing an integrated perspective on complex interventions. J Public Health 2017;39:209-12.

18 Lysdahl KB, Hofmann B. Complex health care interventions: characteristics relevant for ethical analysis in health technology assessment. GMS Health Technol Assess 2016;12:Doc01.

19 May CR, Finch T, Ballini L, et al. Evaluating complex interventions and health technologies using normalization process theory: development of a simplified approach and web-enabled toolkit. BMC Health Serv Res 2011;11:245.

20 Gallio D, Berto P. Health technology assessment (HTA): definition, role and use in the changing healthcare environment. Eur Ann Allergy Clin Immunol 2007;39:7-11.

21 Oortwijn W, Broos $\mathrm{P}$, Vondeling $\mathrm{H}$, et al. Mapping of health technology assessment in selected countries. Int J Technol Assess Health Care 2013;29:424-34.

22 Escafit M, Foy C, Paglia R. The introduction of health technology assessment in Japan; lessons learned from three other new HTa markets (Brazil, Poland and South Korea). Value in Health 2018;21:S5-6.

23 Seo H-J, Park JJ, Lee SH. A systematic review on current status of health technology reassessment: insights for South Korea. Health Res Policy Syst 2016;14:82.

24 Teerawattananon Y, Rattanavipapong W, Lin LW, et al. Landscape analysis of health technology assessment (HTA): systems and practices in Asia. Int $J$ Technol Assess Health Care 2019;35:416-21.

25 Surgey G, Chalkidou K, Reuben W, et al. Introducing health technology assessment in Tanzania. Int J Technol Assess Health Care 2020;36:1-7.

26 Pichon-Riviere A, Soto NC, Augustovski FA, et al. Health technology assessment for decision making in Latin America: good practice principles. Int $J$ Technol Assess Health Care 2018;34:241-7.

27 Luce B, Cohen RS. Health technology assessment in the United States. Int J Technol Assess Health Care 2009;25:33-41.

28 Pfadenhauer L, Rohwer A, Burns J, et al. Guidance for the assessment of context and implementation in health technology assessments ( $\mathrm{HTa}$ ) and systematic reviews of complex interventions: the context and implementation of complex interventions $(\mathrm{CICl})$ framework 2016.

29 Lysdahl KB, Mozygemba K, Burns J, et al. Comprehensive assessment of complex technologies: integrating various aspects in health technology assessment. Int $J$ Technol Assess Health Care 2017;33:570-6.

30 Sorenson C, Drummond M, Kanavos P. Ensuring value for money in health care: the role of health technology assessment in the European Union. Observatory studies series no 11. Copenhagen, Denmark: World Health Organisation on behalf of the the European Observatory on Health Systems and Policies, 2008.

31 O'Rourke B, Oortwijn W, Schuller T, et al. The new definition of health technology assessment: a milestone in international collaboration. Int J Technol Assess Health Care 2020;36:187-90.

32 World Health Organisation. Who International classification for health interventions (ICHI): who, 2019. Available: https://www.who.int/ classifications/ichi/en/ [Accessed 16 May 2019].

33 HTA Glossary. Health technology online: HTa glossary, 2019. Available: http://htaglossary.net/health+technology [Accessed 16 May 2019].

34 Craig P, Dieppe P, Macintyre S, et al. Developing and evaluating complex interventions: new guidance. The UK: Medical Research Council, 2019.

35 Petticrew M, Knai C, Thomas J, et al. Implications of a complexity perspective for systematic reviews and Guideline development in health decision making. BMJ Glob Health 2019;4:e000899.

36 Martín Cantera C, Puigdomènech E, Ballvé JL, et al. Effectiveness of multicomponent interventions in primary healthcare settings to promote continuous smoking cessation in adults: a systematic review. BMJ Open 2015;5:e008807.

37 Guise J-M, Butler ME, Chang C, et al. AHRQ series on complex intervention systematic reviews-paper 6: PRISMA-Cl extension statement and checklist. J Clin Epidemiol 2017:90:43-50.
38 Lorenc T, Felix L, Petticrew M, et al. Meta-Analysis, complexity, and heterogeneity: a qualitative interview study of researchers' methodological values and practices. Syst Rev 2016;5:192.

39 Schildmann J, Nadolny S, Haltaufderheide J, et al. Do we understand the intervention? what complex intervention research can teach us for the evaluation of clinical ethics support services (CESS). BMC Med Ethics 2019;20:48.

40 Ramaswamy R, Reed J, Livesley N, et al. Unpacking the black box of improvement. Int J Qual Health Care 2018;30:15-19.

41 Baghbanian A. Adaptive decision-making and complexity in the Australian healthcare system [PhD]. Sydney, Australia: The University of Sydney, 2010.

42 Baghbanian A, Torkfar G. Economics and resourcing of complex healthcare systems. Aust Health Rev 2012;36:394-400.

43 Snowden DJ, Boone ME. A leader's framework for decision making. A leader's framework for decision making. Harv Bus Rev 2007:85:68-76.

44 Baghbanian A. The emergence of adaptive decision-making in complex health systems. economics and Resourcing of complex health systems: the emergence of adaptive decision-making in health care. Saarbrücken, Germany: LAP Lambert Academic Publishing/ VDM Publishing, 2011.

45 Baghbanian A, Torkfar G, Baghbanian Y. Decision-Making in Australia's Healthcare System and Insights From Complex Adaptive Systems Theory. Journal of Health Scope 2012;1:29-38.

46 Tsiachristas A, Stein KV, Evers S, et al. Performing economic evaluation of integrated care: highway to hell or Stairway to heaven? Int J Integr Care 2016;16:3.

47 Steele Gray C, Shaw J. From summative to developmental: incorporating design-thinking into evaluations of complex interventions. Journal of Integrated Care 2019;27:241-8.

48 Scheirer MA. Linking sustainability research to intervention types. Am $J$ Public Health 2013;103:e73-80.

49 Glasgow RE, Phillips SM, Sanchez MA. Implementation science approaches for integrating eHealth research into practice and policy. Int J Med Inform 2014;83:e1-11.

50 Beletsi A, Koutrafouri V, Karampli E, et al. Comparing use of health technology assessment in pharmaceutical policy among earlier and more recent Adopters in the European Union. Value Health Reg Issues 2018:16:81-91.

51 Kelly MP, Noyes J, Kane RL, et al. AHRQ series on complex intervention systematic reviews-paper 2: defining complexity, formulating scope, and questions. J Clin Epidemiol 2017;90:11-18.

52 Taylor R. What is health technology assessment? UK: Hayward Medical Communications, Hayward Group Ltd, 2009: 6.

53 Jönsson B. Technology assessment for new oncology drugs. Clin Cancer Res 2013;19:6-11.

54 Baghbanian A, Hughes I, Kebriaei A, et al. Adaptive decision-making: how Australian healthcare managers decide. Aust Health Rev 2012;36:49-56.

55 Angelis A, Kanavos P. Multiple criteria decision analysis (MCDA) for evaluating new medicines in health technology assessment and beyond: the advance value framework. Soc Sci Med 2017;188:137-56 https://doi.org/

56 Oortwijn W, Determann D, Schiffers K, et al. Towards integrated health technology assessment for improving decision making in selected countries. Value Health 2017;20:1121-30.

57 Aissaoui A, Soualmi R, Kaddar M. Challenges: in implementing HTa in the reimbursement decisions in Algeria / a Compartive analysis. Value Health 2014;17:A412.

58 Booth A, Noyes J, Flemming K, et al. Formulating questions to explore complex interventions within qualitative evidence synthesis. BMJ Glob Health 2019;4:e001107.

59 Grant MJ, Booth A. A typology of reviews: an analysis of 14 review types and associated methodologies. Health Info Libr J 2009;26:91-108.

60 Peters MDJ, Godfrey C, Mclnerney P, et al. Scoping reviews. In: Aromataris E, Munn Z, eds. Joanna Briggs Institute Reviewer's Manual. Australia: The Joanna Briggs Institute, 2017.

61 Levac D, Colquhoun H, O'Brien KK. Scoping studies: advancing the methodology. Implement Sci 2010;5:69.

62 Arksey H, O'Malley L. Scoping studies: towards a methodological framework. Int J Soc Res Methodol 2005;8:19-32.

63 Peters MDJ, Godfrey CM, Khalil H, et al. Guidance for conducting systematic scoping reviews. Int J Evid Based Healthc 2015;13:141-6.

64 Pham MT, Rajic A, Greig JD, et al. A scoping review of scoping reviews: advancing the approach and enhancing the consistency. Res Synth Methods 2014;5:371-85. 
65 Colquhoun HL, Levac D, O'Brien KK, et al. Scoping reviews: time for clarity in definition, methods, and reporting. J Clin Epidemiol 2014;67:1291-4.

66 Tricco AC, Lillie E, Zarin W, et al. PRISMA extension for scoping reviews (PRISMA-ScR): checklist and explanation. Ann Intern Med 2018;169:467-73.

67 Mays N, Roberts E, Popay J, et al. Synthesising research evidence. In: Allen P, Black N, Clarke A, et al, eds. Studying the organisation and delivery of health services: research methods. London: Routledge, 2001: 188-220.

68 Mathes T, Willms G, Polus S, et al. Health technology assessment of public health interventions: an analysis of characteristics and comparison of methods-study protocol. Syst Rev 2018;7:79.

69 FP7-HEALTH. Final Report Summary - INTEGRATE-HTA (Integrated health technology assessment for evaluating complex technologies): EU Publications Office, 2015. Available: https:// cordis.europa.eu/project/rcn/105703/reporting/es [Accessed 03 Dec 2019].

70 Munn Z, Peters MDJ, Stern C, et al. Systematic review or scoping review? guidance for authors when choosing between a systematic or scoping review approach. BMC Med Res Methodol 2018;18:143.

71 Sucharew H, Macaluso M. Progress notes: methods for research evidence synthesis: the scoping review approach. J Hosp Med 2019;14:416-8.

72 Ghalibaf AK, Nazari E, Gholian-Aval M, et al. Comprehensive overview of computer-based health information tailoring: a scoping review protocol. BMJ Open 2017;7:e019215.

73 Higgins JP, Green S. Cochrane Handbook for systematic reviews of interventions: the Cochrane collaboration 2011.

74 The Joanna Briggs Institute. JBI database of systematic reviews and implementation reports: the Joanna Briggs Institute, 2019. Available: http://journals.Iww.com/jbisrir/pages/default.aspx [Accessed 17 Sep 2019].

75 Joanna Briggs Institute (JBI). Joanna Briggs Institute Reviewers' Manual: 2015 edition/supplement: Methodology for JBI Scoping Reviews. Adelaide, Australia: Joanna Briggs Institute, The University of Adelaide, 2015.

76 McGowan J, Sampson M, Salzwedel DM, et al. PRESS Peer Review of Electronic Search Strategies: 2015 Guideline Statement. J Clin Epidemiol 2016;75:40-6.

77 Merlin T, Tamblyn D, Ellery B, et al. What's in a name? developing definitions for common health technology assessment product types of the International network of agencies for health technology assessment (inahta). Int J Technol Assess Health Care 2014;30:430-7.

78 Dahabreh IJ, Chan JA, Earley A, et al. Modeling and Simulation in the Context of Health Technology Assessment: Review of Existing Guidance, Future Research Needs, and Validity Assessment (Methods Research Report). Rockville, MD: Tufts Evidence-based Practice Center under Contract No. 290-2007-10055-I. AHRQ Publication No.16(17)-EHC020-EF. Agency for Healthcare Research and Quality 2017.
79 Minary L, Trompette J, Kivits J, et al. Which design to evaluate complex interventions? toward a methodological framework through a systematic review. BMC Med Res Methodol 2019;19:92.

80 Daudt HML, van Mossel C, Scott SJ. Enhancing the scoping study methodology: a large, inter-professional team's experience with Arksey and O'Malley's framework. BMC Med Res Methodol 2013;13:48.

81 Armstrong R, Hall BJ, Doyle J, et al. Cochrane Update. 'Scoping the scope' of a cochrane review. J Public Health 2011;33:147-50.

82 Archibald D, Patterson R, Haraldsdottir E, et al. Mapping the progress and impacts of public health approaches to palliative care: a scoping review protocol. BMJ Open 2016;6:e012058.

83 Bragazzi NL, Dini G, Parodi V, et al. Protocol of a scoping review assessing injury rates and their determinants among healthcare workers in Western countries. BMJ Open 2019;9:e023372.

84 Kuupiel D, Bawontuo V, Mashamba-Thompson TP. Mapping evidence on tuberculosis active case finding policies, strategies, and interventions for tuberculosis key populations: a systematic scoping review protocol. Syst Rev 2019;8:162.

85 Halas G, Schultz ASH, Rothney J, et al. A scoping review protocol to map the research foci trends in tobacco control over the last decade. BMJ Open 2015;5:e006643.

86 Chola M, Hlongwana K, Ginindza TG. Mapping evidence on decision-making on contraceptive use among adolescents: a scoping review protocol. Syst Rev 2018;7:201.

87 Braun V, Clarke V. Reflecting on reflexive thematic analysis. Qual Res Sport Exerc Health 2019;11:589-97.

88 QSR International. NVIvo 12 pro Australia: QSR international Pty. LTD, 2019. Available: https://www.qsrinternational.com/nvivo/nvivoproducts/nvivo-12-pro [Accessed 16 Sep 2019].

89 Rodgers C, Rogerson D, Stevenson J, et al. Physical activity for people with young-onset dementia and carers: protocol for a scoping review. Syst Rev 2018;7:36.

90 Miake-Lye IM, Hempel S, Shanman R, et al. What is an evidence MAP? A systematic review of published evidence maps and their definitions, methods, and products. Syst Rev 2016;5:28.

91 Lehoux P, Blume S. Technology assessment and the sociopolitics of health technologies. J Health Polit Policy Law 2000;25:1083-120.

92 Viceconti A, Camerone EM, Luzzi D, et al. Explicit and implicit experience of own's body in painful musculoskeletal disorders and rheumatic diseases: a scoping review protocol of available quantitative and qualitative evidence. SAGE Open Med 2018;6:2050312118820026.

93 Neimann Rasmussen L, Montgomery P. The prevalence of and factors associated with inclusion of non-English language studies in Campbell systematic reviews: a survey and meta-epidemiological study. Syst Rev 2018;7:129.

94 Nussbaumer-Streit B, Klerings I, Dobrescu Al, et al. Excluding non-English publications from evidence-syntheses did not change conclusions: a meta-epidemiological study. J Clin Epidemiol 2020;118:42-54. 